Journal Club

Editor's Note: These short, critical reviews of recent papers in the Journal, written exclusively by graduate students or postdoctoral fellows, are intended to summarize the important findings of the paper and provide additional insight and commentary. For more information on the format and purpose of the Journal Club, please see http://www.jneurosci.org/misc/ifa_features.shtml.

\title{
Contribution of Postsynaptic Molecules to AMPA Receptor Nanodomain Organization
}

\author{
Alvaro Barrera-Ocampo and Thomas E. Chater \\ Laboratory of Synaptic Plasticity and Connectivity, RIKEN Brain Science Institute, Wako-shi, Saitama 351-0198, Japan \\ Review of Nair et al.
}

In the postsynaptic compartment of glutamatergic synapses is an electron-dense region called the postsynaptic density (PSD). The PSD is an organized and dynamic structure that varies in size between synapses and can be modulated by synaptic activity. The PSD comprises $>490$ proteins, including cytoskeletal proteins (e.g., Actin, Tubulin, MAP2), signaling molecules (e.g., CaMKII, PKC $\gamma$, PP2A), scaffolding proteins (e.g., PSD95, GKAP, Shank, Homer), adhesion molecules (e.g., Cadherins, Catenins, Integrins, Neurexin/Neuroligin, NCAM-140), and neurotransmitter receptors (e.g., NMDAR, AMPAR, mGlu1) (Sheng and Hoogenraad, 2007; Chua et al., 2010). The PSD has multiple roles, including controlling synaptic structure and intracellular signaling within a subdomain of the postsynaptic compartment, and it is the primary route through which receptor activation leads to downstream signaling at synapses. Changes in the composition of surface receptors and other PSD elements underlie many forms of synaptic plasticity (Sheng and Hoogenraad, 2007).

The majority of fast excitatory neurotransmission is mediated by heteromeric AMPA receptors (AMPARs), which are composed of four subunits (GluA1-

Received Oct. 6, 2013; revised Nov. 1, 2013; accepted Nov. 5, 2013. We thank Dr. Yukiko Goda for the comments on the manuscript. The authors declare no competing financial interests.

Correspondence should be addressed to Thomas E. Chater, Laboratory of Synaptic Plasticity and Connectivity, RIKEN Brain Science Institute, 2-1 Hirosawa, Wako-shi, Saitama 351-0198, Japan. E-mail: t.e.chater@brain.riken.jp. DOI:10.1523/JNEUROSCI.4273-13.2013

Copyright $\odot 2013$ the authors $\quad 0270-6474 / 13 / 3319048-03 \$ 15.00 / 0$
GluA4) that have different expression patterns across the brain. In the mature neocortex, the most abundant subunits are GluA1 and GluA2, followed by lower levels of GluA3. The subunit composition of AMPARs affects receptor trafficking, kinetics, and calcium permeability (Traynelis et al., 2010). AMPAR subunit composition also affects the diffusion of AMPARs in the plasma membrane, as demonstrated by experiments labeling individual AMPAR subunits at the surface of hippocampal neurons in which GluA2/GluA3 receptors showed increased lateral mobility compared with GluA1/GluA2 receptors at the synapse (Kropf et al., 2008). Although extrasynaptic GluA2-containing AMPARs display high mobility (close to free Brownian motion), when the receptors transit into synapses, their diffusion is slowed, and they may even become immobilized for periods of time (Giannone et al., 2010).

AMPARs interact with multiple auxiliary and scaffolding proteins, which influence the trafficking and stabilization of the receptors at synapses (Fig. 1). The transmembrane AMPAR auxiliary protein (TARP) family links AMPARs to PSD95, thus regulating AMPAR kinetics and location at the PSD. Ultrastructural analysis using immunolabeling electron microscopic tomography showed that PSD95 molecules form filaments that are oriented vertical to the postsynaptic membrane (Chen et al., 2008) and are in contact with horizontal filaments composed of the PSD95-binding partners GKAP and Shank (Chen et al., 2011). The retention of AMPARs at the PSD requires the formation of the complex between PSD95, GKAP, and Shank, as well as interaction with the actin cytoskeleton via Cortactin and other intermediates (Fig. 1). The importance of this interaction for the molecular organization of the PSD was demonstrated by experiments in which Actin depolymerization with Latrunculin A caused dissociation and redistribution of synaptic fractions containing PSD95, GKAP, Shank, and Homer, while the rest of fractions were resistant to the Actin disassembly and remained stably associated with the PSD (Kuriu et al., 2006).

Recent evidence suggests that even finer levels of organization exist within synapses. Using super-resolution light microscopy in live cells, MacGillavry et al. (2013) demonstrated that components of the PSD are organized into higher density clusters within an area $\sim 80 \mathrm{~nm}$ in diameter and that the composition and distribution of these PSD clusters varies substantially between synapses and is affected by changes in synaptic activity. The distribution of scaffolding proteins in individual PSDs is heterogeneous, with proteins like PSD95 forming subsynaptic clusters to capture molecules in transit, as revealed by experiments in which PSD95dense domains were enriched in GluA2containing AMPARs (MacGillavry et al., 2013).

Similarly, Nair et al. (2013) measured levels of AMPAR confinement at synapses that match in size the previously reported PSD95 nanodomains. They used four different super-resolution light imaging methods and pre-embedding immunogold 


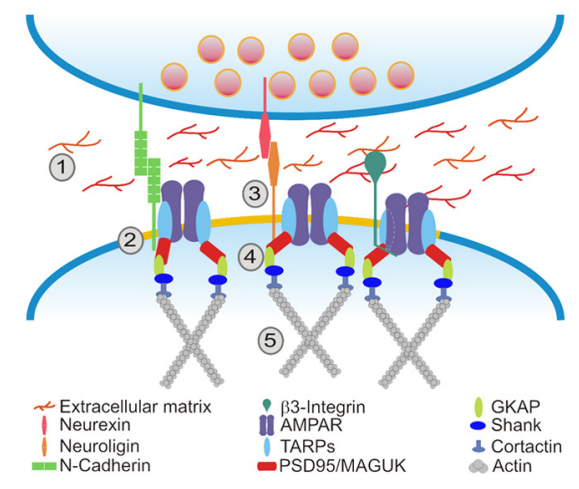

Figure 1. Model of AMPAR nanodomains and the organization levels in the PSD. 1, Interactions between extracellular matrix components and membrane proteins modulate receptor activation and additionally might increase stability by limiting diffusion of proteins into or out of the PSD. 2, The postsynaptic membrane and its lipid composition may regulate the flow of molecules across the synaptic face. 3, Transmembrane and auxiliary proteins stabilize receptors at determined locations in the PSD. 4, Scaffolding proteins connect membrane proteins and signaling molecules with Actin. 5, The cytoskeleton supports the synaptic structure.

electron microscopy to investigate the dynamic organization, lifetime, and stability of AMPAR nanodomains in the synapse. This imaging approach allowed precise measurements of the behavior of AMPARs in live neurons.

The specific labeling of endogenous GluA2 subunits used in the uPAINT (Universal Point Accumulation Imaging in Nanoscale Topography) method allowed visualization of GluA2-containing AMPAR clusters in synaptic spines, and this was confirmed using stimulated emission depletion (STED) microscopy. According to the most accepted AMPAR trafficking model, receptors containing GluA2/GluA3 heteromers are delivered directly to the PSD of dendritic spines, whereas GluA1/GluA2 receptors are delivered to extrasynaptic regions and then diffuse to the PSD after synaptic activity (Passafaro et al., 2001). Using sptPALM (single-particle tracking PhotoActivated Localization Microscopy), Nair and colleagues (2013) observed that coexpression of GluA1 and Eos-tagged GluA2 reduced the diffusion within spines and favored the confinement of GluA2 subunits in dendritic spines, thus demonstrating the significance of the GluAl subunit in the subsynaptic organization of AMPARs.

After AMPARs are delivered to the postsynaptic membrane, auxiliary proteins like stargazin anchor the receptors to scaffolding proteins, helping to locate them within the PSD to form nanodomains. Nair et al. (2013) analyzed the contribution of stargazin to the organization of AMPAR nanodomains and observed that disrupting the association between stargazin and PSD95 (by overexpressing the stargazin mutant StgT231F) reduced the density of AMPAR nanodomains but increased their size, and AMPAR mobility neuron-wide.

In additional experiments, Nair et al. (2013) used live-labeling and immunogold electron microscopy to confirm the size and location of the AMPAR clusters. They established that clusters within spines had an average length of $\sim 77 \mathrm{~nm}$ and were located in the PSD ( $\sim 2$ nanodomains) or extrasynaptic regions ( $\sim 4$ nanodomains). Analysis of the movement of endogenous GluA2containing AMPARs between nanodomains identified three patterns: Type 1 receptors moved in/out of the nanodomain, suggesting they did not engage in stabilizing interaction with other proteins; Type 2 receptors moved in and out of nanodomain, but spent more time inside; and Type 3 receptors were confined inside nanodomains, reflecting stable associations with interacting proteins. The lifetime of AMPAR nanodomains varied significantly, with $40 \%$ of nanodomains lasting $<5 \mathrm{~min}$, and the remaining $60 \%$ sometimes remaining stable for up to $45 \mathrm{~min}$, demonstrating the existence of subtypes of nanodomains.

The dynamics of AMPARs and their stabilization within nanodomains seems to be a tightly controlled process involving different levels of interaction and organization. To shed light on this aspect, Nair et al. (2013) evaluated the contribution of PSD95 to the organization of AMPAR nanodomains by expressing Eos-tagged PSD95 and labeling endogenous GluA1 in neurons. They confirmed the existence of PSD95 clusters $\sim 424 \mathrm{~nm}$ in size within the PSD, and found that these clusters partially colocalized with AMPAR nanodomains. In contrast, a previous study reported a high degree of colocalization between AMPARs and PSD95 (MacGillavry et al., 2013). This disagreement may be the result of overexpressing both proteins and the lack of direct interaction between AMPAR and PSD95. However, Nair and colleagues (2013) clarified the role of PSD95 as a fundamental organizer of AMPAR nanodomains by modulating the levels of PSD95 in neurons. Knock-down of PSD95 decreased the number of AMPAR nanodomains/spine and increased the mobility of receptors inside synapses, whereas overexpression of PSD95 caused the opposite effect. Using dSTORM, the authors evaluated the impact of PSD95 modulation on the number of AMPARs/ nanodomain and observed a reduction of $21 \%$ in the number of receptors when PSD95 was knocked-down, whereas overexpression did not alter the amount of AM-
PARs. As a consequence of the reduced levels of PSD95 and AMPARs/nanodomain, the amplitude of miniature EPSCs was decreased by $20 \%$. These results agree with simulations that suggest that lower density of AMPARs and distances $>100 \mathrm{~nm}$ from the glutamate release site decrease the amplitude of mEPSCs, thus emphasizing the importance of specific positioning of AMPARs in synaptic transmission. These data suggest a novel way to potentiate synaptic transmission that does not rely on receptor trafficking or phosphorylation, and instead requires the movement of clustered receptors so they sit directly opposite release sites. Both the modeling data from MacGillavry et al. (2013) and the mEPSC recording from Nair et al. (2013) support this hypothesis.

It is clear that the localization of AMPARs in the PSD involves the recruitment of several molecules acting as organizers at different structural levels of the postsynaptic terminal. It would be interesting to see whether other synaptic organizers are themselves clustered into nanodomains and/or contribute to the transit, lateral diffusion, and organization of AMPARs. For example, many adhesion proteins, such as $\mathrm{N}$-Cadherin, $\beta 3$-Integrin, and Neurexin-1/ Neuroligin-1, are present at the synapse and can interact with AMPARs (Fig. 1). AMPARs interact with $\mathrm{N}$-Cadherin through the N-terminal domain of GluA2, which slows its synaptic diffusion (Saglietti et al., 2007). This may influence the positioning of AMPARs within the PSD and help align the receptors with the neurotransmitter release site in the active zone, which would strongly potentiate the postsynaptic response to release events. Interestingly, GluA2 also interacts with $\beta 3$-Integrin by means of the cytoplasmic domain of both proteins. The binding stabilizes AMPARs at the surface of synapses. $\beta 3$-Integrin lacks a PDZ domain, precluding direct PSD95 binding; however, its ability to bind the extracellular matrix hints at another possible way of localizing AMPARs at synaptic sites (Pozo et al., 2012). Unlike N-Cadherin and $\beta 3$ Integrin, Neurexin-1/Neuroligin-1 does not interact directly with AMPARs. Rather, Neuroligin-1 associates with PSD95 to trap GluA2-containing AMPARs at nascent synapses. The functional relevance of this confinement was demonstrated using hippocampal slices of Neuroligin-1 knockout mice, which displayed reduced levels of synaptic AMPARs and AMPAR-mediated synaptic transmission (Mondin et al., 2011). Trans-synaptic signaling via adhesion proteins could potentially synchronize changes 
in postsynaptic receptor distribution and presynaptic release probability.

The existence of nanodomains demonstrates that there is a high degree of spatial organization within synapses, and that proteins are heterogeneously distributed, at least on the postsynaptic side of the cleft. Functionally, this clustering of various PSD molecules (receptors, scaffolds, and downstream effectors) could enable the fine control of synaptic signaling by bringing proteins into close proximity to each other. Whether presynaptic active zones mirror postsynaptic receptor/PSD nanodomain clusters remains to be shown.

\section{References}

Chen X, Winters C, Azzam R, Li X, Galbraith JA, Leapman RD, Reese TS (2008) Organization of the core structure of the postsynaptic density. Proc Natl Acad Sci U S A 105:4453-4458. CrossRef Medline

Chen X, Nelson CD, Li X, Winters CA, Azzam R, Sousa AA, Leapman RD, Gainer H, Sheng M, Reese TS (2011) PSD-95 is required to sustain the molecular organization of the postsynaptic density. J Neurosci 31:6329-6338. CrossRef Medline

Chua JJ, Kindler S, Boyken J, Jahn R (2010) The architecture of an excitatory synapse. J Cell Sci 123:819-823. CrossRef Medline

Giannone G, Hosy E, Levet F, Constals A, Schulze K, Sobolevsky AI, Rosconi MP, Gouaux E, Tampé R, Choquet D, Cognet L (2010) Dynamic superresolution imaging of endogenous proteins on living cells at ultra-high density. Biophys J 99:1303-1310. CrossRef Medline

Kropf M, Rey G, Glauser L, Kulangara K, Johnsson K, Hirling H (2008) Subunit-specific surface mobility of differentially labeled AMPA receptor subunits. Eur J Cell Biol 87: 763-778. CrossRef Medline

Kuriu $\mathrm{T}$, Inoue A, Bito $\mathrm{H}$, Sobue K, Okabe S (2006) Differential control of postsynaptic density scaffolds via actin-dependent and -independent mechanisms. J Neurosci 26: 7693-7706. CrossRef Medline

MacGillavry HD, Song Y, Raghavachari S, Blanpied TA (2013) Nanoscale scaffolding domains within the postsynaptic density concentrate synaptic AMPA receptors. Neuron 78:615-622. CrossRef Medline

Mondin M, Labrousse V, Hosy E, Heine M, Tessier B, Levet F, Poujol C, Blanchet C, Choquet D, Thoumine O (2011) Neurexin-neuroligin adhesions capture surface-diffusing AMPA receptors through PSD-95 scaffolds. J Neurosci 31: 13500-13515. CrossRef Medline

Nair D, Hosy E, Petersen JD, Constals A, Giannone G, Choquet D, Sibarita JB (2013) Super- resolution imaging reveals that AMPA receptors inside synapses are dynamically organized in nanodomains regulated by PSD95. J Neurosci 33:13204-13224. CrossRef Medline

Passafaro M, Piëch V, Sheng M (2001) Subunitspecific temporal and spatial patterns of AMPA receptor exocytosis in hippocampal neurons. Nat Neurosci 4:917-926. CrossRef Medline

Pozo K, Cingolani LA, Bassani S, Laurent F, Passafaro M, Goda Y (2012) beta3 integrin interacts directly with GluA2 AMPA receptor subunit and regulates AMPA receptor expression in hippocampal neurons. Proc Natl Acad Sci U S A 109:1323-1328. CrossRef Medline

Saglietti L, Dequidt C, Kamieniarz K, Rousset MC, Valnegri P, Thoumine O, Beretta F, Fagni L, Choquet D, Sala C, Sheng M, Passafaro M (2007) Extracellular interactions between GluR2 and N-cadherin in spine regulation. Neuron 54:461-477. CrossRef Medline

Sheng M, Hoogenraad CC (2007) The postsynaptic architecture of excitatory synapses: a more quantitative view. Annu Rev Biochem 76:823-847. CrossRef Medline

Traynelis SF, Wollmuth LP, McBain CJ, Menniti FS, Vance KM, Ogden KK, Hansen KB, Yuan H, Myers SJ, Dingledine R (2010) Glutamate receptor ion channels: structure, regulation, and function. Pharmacol Rev 62:405-496. CrossRef Medline 